\title{
Del discurso a las prácticas: Políticas sociales y psicología comunitaria en Chile
}

\author{
María Isabel Reyes Espejo \\ Universidad Santo Tomás. Santiago, Chile. \\ Email:mreyese@santotomas.cl
}

Bárbara Olivares Espinoza

Universidad Diego Portales. Santiago, Chile.

Email: barbara.olivares@mail.udp.cl

\section{Héctor Berroeta Torres}

Universidad de Valparaíso. Valparaíso, Chile.

Email: hector.berroeta@uv.cl

\section{María Inés Winkler Müller}

Universidad de Santiago de Chile. Santiago, Chile.

Email: maria.winkler@usach.cl

Resumen: ${ }^{1}$ En este trabajo se aborda descriptivamente las dimensiones ética y política de la Psicología Comunitaria (PC) en la política social chilena a partir de un estudio cualitativo que contempló tres fases progresivas de estudio: (1) Confección de un catastro de la oferta de programas comunitarios (PCo) vigentes en los sitios web de diez Ministerios ( $\mathrm{N}=101$ ); (2) Análisis descriptivo de las orientaciones y prácticas implementadas en PCo cercanos a los principios ético-políticos de la PC; y (3) Estudio de caso de tres PCo ejecutados en la Región de Valparaíso y Metropolitana. Los resultados muestran una escasa convergencia entre los principios y valores ético-políticos de la PC y los fundamentos y prácticas implementadas en los PCo examinados, mientras que a nivel metodológico existe mayor cercanía con modelos y estrategias propias de la disciplina. Se concluye que la incidencia de la PC chilena en las políticas sociales se limita sólo al plano técnicometodológico, excluyendo sus principios orientadores.

Palabras clave: política social, psicología comunitaria, intervención comunitaria, programas sociales, Chile.

\section{From the discourse to the practices: Social policy and community psychology in Chile}

\begin{abstract}
In this work we address through a descriptive approach the ethical and political dimensions of Community Psychology (CP) in Chilean social policy, starting from a qualitative study which implied three progressive phases: (1) Confection of a cadastre on the offer of Community Programs (CPr) currently
\end{abstract}


available on websites of ten Ministries ( $\mathrm{N}=101)$; (2) Descriptive analysis of the orientations and practices implemented in CPr close to ethical-political principles of CP; and (3) Case study of three CPr executed in the Valparaiso and Metropolitan regions. The results show a poor convergence between the principles and ethicalpolitical values of CP and the foundations and practices implemented in examined PCr, while at methodological level exists greater closeness models and strategies proper to the discipline. It is concluded that the incidence of Chilean's CP in social policies is limited only to technical-methodological level, excluding its guiding principles.

Keywords: social policy, community psychology, community intervention, social programs, Chile.

\section{Do discurso para as práticas: as políticas sociais e de psicologia comunitária no Chile}

Resumo: Este trabalho trata descritivamente as dimensões éticas e políticas da Psicologia Comunitária (PC) na política social chilena mediante um estudo qualitativo que analisou três fases progressivas de estudo: (1) Elaboração de um inventário da oferta de programas comunitários (PCo) existentes em sites de dez Ministérios ( $\mathrm{N}=101)$; (2) A análise descritiva das orientações e práticas implementadas em PCo próximos dos princípios éticos e políticos da PC; e (3) Estudo de caso de três PCo executados na região de Valparaíso e Metropolitana. Os resultados mostram pouca convergência entre os princípios e valores ético-políticos da PC e os fundamentos e práticas implementadas nos PCo examinados, enquanto que em nível metodológico existe maior aproximação com modelos e estratégias próprias da disciplina. Conclui-se que a incidência da PC chilena nas políticas sociais limita-se apenas ao nível técnico e metodológico, excluindo seus princípios orientadores.

Palavras-chave: Política Social, Psicologia Comunitária, Intervenção comunitária, programas sociais, Chile.

$$
* * *
$$

\section{Psicología Comunitaria y Políticas Públicas: una relación compleja}

Actualmente, la PC chilena experimenta un momento de expansión y reconocimiento, caracterizado por la ampliación del campo de inserción profesional para los/as psicólogos/as a través de la creación de nichos para la formación en PC, mediante cursos y menciones en pregrado y maestrías en posgrado, junto con la generación de nuevas plazas laborales, principalmente en programas derivados de políticas públicas (Alfaro \& Zambrano, 2009; Berroeta, Hatibovic \& Asún, 2012; Alfaro, 2013; Berroeta, 2014). Distintos/as autores/as reportan que, paradójicamente, este proceso ha traído también importantes dificultades en el quehacer profesional, llegando a poner "en jaque" la calidad de las intervenciones (Saavedra, 2005; Berroeta, 2014) y situando a los/as psicólogos/as como técnicos/as, en un entorno laboral precario e inespecífico (Winkler, Alvear, Olivares \& Pasmanik, 2012). Estas constataciones fundamentan la necesidad de conocer, describir y 
analizar las formas en que se incorpora la perspectiva comunitaria en un conjunto de programas gubernamentales en Chile que se declaran "comunitarios" en tanto, estos son los espacios donde se despliega el ejercicio profesional de la disciplina y se concretan sus aportes teórico-prácticos.

En diversos contextos y la latitudes se ha planteado un vínculo histórico y de influencia mutua entre la PC y las políticas sociales (Alfaro, 2013). En Chile, esta vinculación tiene una larga data, pues desde los años sesenta los/as psicólogos/as, junto a otros/as profesionales, han sido parte de la ejecución de programas sociales. Sin embargo, a partir de la década de los ochenta, estas prácticas establecen una relación explícita y directa con la PC, siendo los noventa un momento clave, cuando la PC avanza en su consolidación como disciplina a partir de la implementación de políticas públicas que, en su mayoría, buscaban superar la pobreza que afectaba al país. En concreto, a principio de los noventa, emergen nuevos temas y prioridades en el enfoque del nuevo Estado democrático que se relacionan fundamentalmente con la pobreza y la desigualdad, optando por una estrategia de focalización selectiva que buscaba intervenir en los denominados "grupos vulnerables", a saber: etnias, mujeres, discapacidad, infancia, juventud, tercera edad. Este abordaje dio origen a nuevos programas e instituciones que hicieron posible la aparición de un campo de acción que desarrolla intervenciones que incorporan procesos psicosociales ligados a la subjetividad y la sociabilidad. Se configura, entonces, un nuevo marco de demandas hacia los equipos técnicos y profesionales, que convoca a psicólogos/as formados/as en PC (Alfaro \& Zambrano, 2009).

Este contexto político favoreció que los/as psicólogos/as insertos/ as en la academia formaran a profesionales que luego se desempeñaron laboralmente en espacios de las nuevas instituciones, políticas públicas, programas sociales y de salud. Su incorporación permitió visibilizar y aplicar conceptos y estrategias de desarrollo que representaban una postura psicosocial que fue adquiriendo relevancia y despertando interés en distintos espacios. Se consolida, así, un enfoque comunitario tanto a nivel del gobierno central como local, impulsando un diálogo intra e intersectorial, donde se vincularon instituciones públicas con organizaciones de la sociedad civil, severamente excluidas del debate público hasta ese momento (Asún \& Unger, 2007).

No obstante, es importante considerar que el modelo socioeconómico neoliberal chileno se constituye en la impronta que delimita y, en algún sentido, sostiene las prácticas de la PC en el contexto actual. En este sentido, algunos autores plantean que bajo la hegemonía del sistema capitalista en el marco de la globalización, la construcción de las políticas sociales ha respondido desde hace varios años a una lógica instrumental y determinista, a partir de la focalización del gasto social y obliterando la construcción de ciudadanía (Delamaza, 2011; Fuenmayor, 2014). Así, en los comienzos del nuevo milenio, el enfoque de la PC se instala en la política pública chilena, desarrollando modelos y técnicas en los distintos dispositivos de intervención, como es la asociatividad y apoyo psicosocial en el Fondo de Solidari- 
dad e Inversión Social [FOSIS]; la intervención en crisis y el enfoque de redes en el Servicio Nacional de Menores [SENAME]; autogestión y participación en el Ministerio de Vivienda Urbana [MINVU], la promoción e inserción social en los programas de salud mental o el empowerment en los programas de seguridad pública (Krause, Jaramillo, Monreal, Carvacho \& Torres, 2011).

Si bien la incorporación de la PC al campo de las políticas sociales ha favorecido su consolidación profesional y académica, los escenarios y condiciones políticas actuales han generado nuevas fronteras y retos para la producción de prácticas comunitarias que es pertinente y relevante revisar. En el marco del sistema neoliberal estadounidense se han documentado lógicas contradictorias entre las propuestas de intervención y organización que promueve la PC y la construcción de políticas sociales; por una parte, se ha observado que una gestión pública basada en la descentralización y privatización de los servicios ha repercutido fuertemente en el tercer sector, coaccionando su potencial crítico y cuestionador del Estado y convirtiéndolo en un "cliente-consumidor" más, sometido a los principios del mercado (Hasenfeld \& Garrow, 2012). Por otra, de acuerdo a Brady, Schoeneman \& Sawyer (2014) el sistema neoliberal ha impactado en la organización comunitaria estadounidense a través de 3 tendencias: 1) la promoción de una práctica basada en la evidencia como un proceso dominante para guiar la acción profesional; 2) la atención disminuida y la tergiversación de los movimientos sociales en el contexto teórico-práctico de la educación de las comunidades y 3) la creciente profesionalización de la organización comunitaria. En este contexto, esta última ha tendido a "depender" fuertemente de prácticas tecno-científicas, centradas en formas "objetivas" de conocimiento que se traducen en intervenciones de corte positivista/cuantitativo, que tienden a mantener el status quo a través de la promoción de reformas menores y fortalecen el individualismo neoliberal (Brady, Schoeneman \& Sawyer, 2014).

En otros países se ha documentado que el aumento de los proyectos de intervención comunitaria no tiene un impacto directo en el fortalecimiento del compromiso político de los/as psicólogos/as sociales, ni ha aumentado la participación de las comunidades involucradas, como describe Fátima Quintal de Freitas (2000) para Brasil. Maritza Montero (2010) identifica como uno de los principales focos de tensión la verticalidad de la relación que establece la política pública con las comunidades en Venezuela. Muchos de los programas públicos son dirigidos desde afuera hacia adentro, o de arriba hacia abajo, sin informar, ni sensibilizar a la comunidad respecto de la solución o cambio propuesto.

En este sentido, la recurrida metáfora de haber "bailado con un monstruo", acuñada por Marybeth Shinn (2007), sintetiza bien la complejidad que describe Montero (2010) para llevar a cabo una intervención comunitaria en programas sociales. Esta autora, a partir del análisis de su experiencia, concluye que hay que asumir que los/as funcionarios/as públicos/as se encuentran permanentemente sujetos/as a presiones y deben responder a 
intereses diversos, por lo que es necesario desarrollar y consolidar relaciones con ellos/as, para que puedan utilizar los conocimientos que se les ofrece desde la PC y estar disponibles cuando el ritmo se los permita. Es necesario reconocer, también, que los conocimientos provenientes de las ciencias sociales no son los únicos a considerar y que muchas veces, no son los que cuentan con mayor legitimidad, por lo que también existirán resistencias.

Sin embargo, a pesar de estas dificultades, la literatura reciente plantea como deseable la contribución mutua entre estos campos. Se señala que la investigación en PC puede aportar a la toma de decisiones en Políticas Sociales, que los procesos de intervención comunitaria contribuyen al diseño de políticas más pertinentes a las necesidades de las comunidades y que la participación de las comunidades mejora el sentido y sostenibilidad de las acciones (Montero, 2009; Ornelas, Vargas \& Duarte, 2010; Rodríguez, 2012; Nelson, 2013; Alfaro, 2013, Wiesenfeld, 2014). Se reconoce, además, una ventaja interesante en este desafío para la disciplina: trabajar con los distintos estamentos gubernamentales pueden mejorar la calidad de la PC, pues este tipo de trabajo requiere de esfuerzos y colaboración, obligando a los/as profesionales a ser creativos/as y estratégicos/as en la formulación de propuestas.

Diversas experiencias muestran cómo la PC ha logrado legitimarse como saber experto en la producción de programas y políticas, siendo un horizonte posible para el desarrollo disciplinar chileno (Alfaro, 2013). Por ejemplo, en Estados Unidos, se ha reportado que existen mecanismos institucionales que promueven la incorporación de psicólogos/as a los procesos de toma de decisiones que dan origen a las políticas públicas. En concreto, anualmente la APA entrega becas a psicólogos/as para que asistan al parlamento y estudien la producción de políticas, con el fin de incidir en ellas y mejorarlas (Lorion, 1996). En Argentina, por su parte, en la provincia de Buenos Aires, se ha creado recientemente la Dirección de PC y Pedagogía Social, que depende de la Dirección General de Cultura y Educación, que es la primera área gubernamental que incorpora la PC en la política pública de educación (Lapalma \& de Lellis, 2012). En Costa Rica se ha valorado la participación de psicólogos/as comunitarios/as en la formulación de nuevas orientaciones en el área de la infancia y adolescencia, que han promovido el desarrollo de una política holística centrada en la defensa de los derechos y en el rol prioritario de la comunidad, dejando atrás un modelo asistencialista centrado en el trabajo sobre aspectos fragmentarios concebidos como indicadores de deterioro social (Arrieta \& Garita, 2012).

En Colombia, dos iniciativas responderían a dicho espíritu; por una parte, la propuesta de una política pública de la convivencia a partir de la creación de una Red de Promoción del Buen Trato y de Prevención de Violencia Intrafamiliar (Arango Cálad, 2006 en Montero 2010) y, por otra, la figura del consorcio social como una forma de organización que cuenta con una base en la comunidad, pero que incorpora a técnicos/as, especialistas y organismos del Estado ejecutores de políticas públicas, con distintos 
niveles de participación y posibilidad de obtener un manejo eficiente y equilibrado de conocimientos, recursos e intereses comunitarios, más beneficios políticos (Montero, 2010).

En otros casos, existe un cierto consenso respecto de la necesidad de que psicólogos/as participen en la producción de política pública; sin embargo, esto aún es incipiente. En Puerto Rico, Carvallo-Messa \& Serrano-García (2008) sugieren fomentar la colaboración entre legisladores/as y psicólogos/as y otros/as científicos/as sociales en sus equipos de trabajo; en los currículos universitarios, incluir formación en política pública y educación continua; crear redes y alianzas con instituciones involucradas en la producción e implementación de política pública, así como con los institutos de política pública a nivel local e internacional y generar actividades comunitarias y municipales en las que los/as psicólogos/as y otros/as científicos/as sociales puedan impactar las políticas públicas.

Creemos importante destacar que muchas de estas experiencias, no han surgido a propósito de decisiones en la gestión pública, sino que más bien ha dependido de esfuerzos personales de equipos técnicos que han utilizado espacios institucionales para instalar enfoques conceptuales y modelos operativos provenientes de la PC (Lapalma \& de Lellis, 2012).

En Chile, a pesar de las dificultades ya descritas, la incorporación de la PC en el diseño de programas públicos ha sido una realidad concreta que ha permitido experiencias auspiciosas que definen un trayecto deseable para la disciplina en su permanente diálogo con las preocupaciones y aspiraciones ciudadanas. De este modo, la PC puede tener un papel importante en potenciar el carácter emancipatorio y la orientación al cambio social en las políticas sociales, contrarrestando la tendencia de la política pública clásica -de corte neoliberal- que se centra en la compensación del déficit y el control social (Alfaro, 2012). Sin embargo, no conocemos reportes sistemáticos de la formas en que tal asociación está ocurriendo en la práctica, lo que nos ha llevado a la formulación del presente trabajo que se propone identificar y revisar en qué medida y cómo se incluye una perspectiva comunitaria en programas derivados de las políticas públicas, o, en palabras de Shinn (2007), conocer algunos de los pasos del “baile con el monstruo”.

\section{Aproximación metodológica}

Hemos optado por un enfoque cualitativo de investigación, dado que éste es concordante epistemológicamente con la PC, particularmente en cuanto a la valoración de la diversidad, del contexto y la importancia de los sujetos sociales, en referencia a su discurso y accionar (Banyard \& Miller, 1998; Stein \& Mankowski, 2004). Enfatizamos el carácter constructivo-interpretativo del proceso de producción científica (González Rey, 2009), mediado por el reconocimiento de procesos situacionales (plano material e histórico) y afectado por las prácticas sociales y el contexto en que éstas se producen (Flores, 2010). 
En base a estas directrices, nos aproximamos a la oferta de programas públicos vigentes en el contexto gubernamental chileno, a partir de la estructuración de un dispositivo de investigación inspirado en los principios de la teoría fundada empíricamente (Glaser \& Strauss, 1967), cuyo objetivo es generar modelos explicativos que se encuentren apoyados en los datos cualitativos y cuantitativos o la combinación de ambos. Esta aproximación contempla diversas fases que se retroalimentan entre si. De este modo, para tratar el corpus textual obtenido a partir las diferentes fuentes de información, empleamos la técnica de análisis de contenido, llevando a cabo codificación abierta y axial, estableciendo inferencias fundadas a partir de ésta (Iñiguez, 1999).

En una primera fase de estudio, se revisaron las páginas web de los 23 ministerios del Gobierno de Chile, durante los meses de Marzo a Mayo de 2013, y confeccionamos un catastro que registra descriptivamente la información de 10 ministerios que por su naturaleza y pertinencia de objetivos, cumplían con el criterio de albergar programas, proyectos y fondos concursables que explicitaban su foco en el campo comunitario y que contenían elementos básicos del encuadre propuesto por la PC. Así, operamos indagando en las formas en que se manifestaba (o no) una perspectiva comunitaria e identificando algunas de sus características a partir de categorías pre-establecidas (dimensiones ético-políticas) que sirvieron de criterios de inclusión para el catastro: 1) Se presentan explícitamente como programas “comunitarios" y 2) En sus objetivos se hace referencia a potenciar dinámicas relacionales (entre sujetos, grupos y/o comunidades) o de desarrollo comunitario.

El trabajo previo nos permitió transitar a una segunda fase de investigación, en la que incorporando dos filtros de selección más, aumentamos la exigencia relativa a lo que podría ser considerado comunitario. En este sentido, los 101 programas catastrados fueron examinados en función de si: 1) Además de plantearse como programas comunitarios, para su ejecución se contemplaba una estrecha vinculación con una/la comunidad (se releva la dimensión territorial) y 2) además de buscar la potenciación y/o desarrollo comunitario, se plantea desde una la acción programática no asistencial. Así, durante los meses de Mayo a Julio de 2012 profundizamos en el estudio de los 9 casos que superaron estos criterios, contemplando analíticamente su fichaje a través de la observación de 9 categorías: 1) Duración, 2) Objetivos generales, 3) Objetivos específicos, 4) Destinatarios/as, 5) Vía de ingreso, 6) Focalización territorial, 7) Elementos del diseño y/o implementación, 8) Presupuesto, 9) Características de los equipos de ejecución. Para la consecución de esta etapa de estudio, consultamos sus páginas web, examinando las bases técnicas junto a una serie de documentos disponibles que fundamentaban sus características, orientación y evaluación. Cabe destacar que en este proceso también debimos recurrir a la búsqueda de información por vía telefónica, personal y electrónica mediante el portal de transparencia (http:// www.portaltransparencia.cl/PortalPdT/). 
Con el propósito de profundizar en las características y el proceso de construcción de la política social en el país en una tercera fase de estudio recurrimos a la metodología del estudio de caso para aproximarnos, durante los meses de Agosto de 2013 a Enero de 2014, a tres programas en ejecución tanto en las regiones Metropolitana y de Valparaíso que lograron superar un tercer filtro, a través de la aplicación de los siguientes criterios: 1) que relevara en sus objetivos una orientación al grupo o la comunidad, pero establecido a nivel territorial a partir de la acción programática desde una perspectiva no asistencial; b) que implicase una vinculación en contexto comunitario, buscando potenciar dinámicas relacionales (entre sujetos, grupos y/o comunidades); c) que estuviese en ejecución, al menos, desde el 2010 y d) que tuviese una duración mínima de 6 meses.

Para acceder al discurso de los actores y procesos, realizamos entrevistas semi-estructuradas a un conjunto de diseñadores/as, ejecutores/as y usuarios/as de los programas, cuyo propósito fue conocer motivaciones, creencias, esquemas de interpretación y modos de significación que los/as entrevistados/as tenían respecto al sentido y alcance de su participación como agentes de cambio en sus diversos campos y contextos (Gaínza, 2006). Además, utilizamos técnicas documentales y textuales para analizar documentos, libros, comunicaciones, páginas web, como productos habituales de los distintos contextos materiales que las producen (Iñiguez, 1999). A continuación, detallamos la caracterización de los/as entrevistados/as y el número de documentos analizados para cada programa.

\section{Tabla N ${ }^{\circ}$ 1: Recolección de Datos por Programa, Participantes y Documentos.}

\begin{tabular}{|c|c|c|}
\hline Programa & Participantes en entrevistas & Documentos \\
\hline $\begin{array}{r}\text { Programa Recuperación de } \\
\text { Barrios }\end{array}$ & $\begin{array}{l}1 \text { diseñador ( } 2 \text { entrevistas) } \\
1 \text { ejecutor ( } 1 \text { entrevista) } \\
2 \text { usuario ( } 2 \text { entrevistas })\end{array}$ & 5 documentos técnicos \\
\hline $\begin{array}{r}\text { Programa Mejoramiento de } \\
\text { atención a la Infancia }\end{array}$ & $\begin{array}{l}2 \text { diseñadores ( } 2 \text { entrevistas) } \\
2 \text { ejecutores ( } 2 \text { entrevistas) } \\
1 \text { usuaria ( } 1 \text { entrevista) }\end{array}$ & 3 documentos técnicos \\
\hline $\begin{array}{r}\text { Programa de Prevención } \\
\text { Comunitaria }\end{array}$ & $\begin{array}{l}2 \text { diseñadores ( } 4 \text { entrevistas) } \\
4 \text { ejecutores ( } 3 \text { entrevistas) } \\
12 \text { usuarios ( } 2 \text { entrevistas } \\
\text { grupales) }\end{array}$ & 5 documentos técnicos \\
\hline TOTAL & $\begin{array}{l}\mathrm{N}=27 \text { participantes } \\
17 \text { entrevistas individuales } \\
2 \text { entrevistas grupales }\end{array}$ & 13 documentos técnicos \\
\hline
\end{tabular}

En su conjunto, esta estrategia de trabajo en etapas, nos permitió una aproximación heurística hacia el fenómeno en estudio, ya que, por un lado, recurrimos a diferentes fuentes de información (recogidas en distintos períodos temporales) y, por otro, se favoreció la confluencia de una serie de técnicas de análisis para aproximarse y caracterizar al fenómeno de estudio (Miles \& Huberman, 1994; Denzin \& Lincoln, 1994). Así, en lo siguiente, reportaremos resultados derivados de dos tipos de perspectivas de análisis: 1) de tipo descriptivo/cuantitativo y 2) de tipo cualitativo. 


\section{La extensión de lo comunitario en la oferta programática política social chilena}

Para concretar una observación sistemática de las características de la política social chilena en el marco de los principios ético-políticos de la psicología comunitaria, optamos por revisar las páginas web de la lista completa de ministerios, seleccionando aquellos que por su naturaleza y pertinencia de objetivos, podían albergar programas con alguna dimensión comunitaria. De los ministerios existentes, seleccionamos una muestra de 10 que cumplían con tal criterio y que, además, podían servir de marco de referencia para examinar las demandas contingentes hacia los equipos técnicos y profesionales. En base a estos antecedentes construimos un catastro que contiene información relativa a los siguientes ministerios: de Desarrollo Social, de Vivienda y Urbanismo, del Interior, del Medio Ambiente, de Educación, de Trabajo y Previsión Social, Secretaría General de Gobierno, de Justicia, de Salud y el Consejo Nacional de la Cultura y las Artes.

Para cada uno de ellos, revisamos el listado total de programas, proyectos y fondos concursables presentes en sus respectivas páginas web, seleccionando aquellos que explicitaban en sus orientaciones programáticas elementos básicos del encuadre comunitario. Con este primer filtro presente, seleccionamos 101 casos considerados "con cercanía a la perspectiva comunitaria”, constituyéndose así en un importante corpus susceptible de examinar con mayor profundidad. A partir de este primer análisis, elaboramos dos criterios que sirvieron de indicadores para evaluar la pertinencia de cada programa/proyecto/fondo previamente descritos en la metodología (filtro 2).

A modo de ejemplo, entregamos a continuación la información extractada y analizada del Ministerio de Desarrollo Social. Con ello ilustramos tanto el tipo de estructura que poseen la mayoría de los ministerios, como la forma en que fuimos catastrando y realizando la selección de programas, con la aplicación de los criterios que nos permitiesen derivar los programas que efectivamente poseen una perspectiva coincidente con los planteamientos de la PC (Ver Tabla $\mathrm{N}^{\circ}$ 2). En primera instancia, revisamos la División de Promoción y Protección Social, donde encontramos el Programa Chile Crece Contigo y el Sistema Chile Solidario que a su vez, contiene al Programa Puente, Programa Caminos, Programa Vínculos y Programa Calle. Es decir, consideramos 5 programas, que bajo un examen de sus orientaciones, alcances y características, no cumplieron los criterios previamente señalados. Inspeccionamos, además, la Oficina de Servicios Relacionados que contiene a la Corporación Nacional de Desarrollo Indígena, (CONADI, con 12 proyectos), el Instituto de la Juventud (INJUV, con 2 proyectos), el Servicio Nacional de la Discapacidad (SENADIS), FOSIS (con 12 proyectos) y el Servicio Nacional del Adulto Mayor (SENAMA, con 7 proyectos). En total, la revisión consideró 33 proyectos de los cuales solamente uno logró responder a todos los criterios: “A Mover Chile” perteneciente al INJUV. 
Tabla No 2: Programas, Proyectos y Fondos del Ministerio de Desarrollo Social en el base a la Subsecretaría de Servicios Sociales y División de Promoción y Protección Social².

\begin{tabular}{|c|c|c|}
\hline Programas/características & Proyectos/Fondos & Criterios \\
\hline \multirow{3}{*}{$\begin{array}{r}\text { Sistema Chile Solidario: } \\
\text { Promueve la incorpora- } \\
\text { ción de familias y } \\
\text { personas en situación de } \\
\text { extrema pobreza a las } \\
\text { redes sociales y acceso a } \\
\text { mejores condiciones de } \\
\text { vida. }\end{array}$} & $\begin{array}{l}\text { Programa Puente: ejecutado por mu- } \\
\text { nicipalidades y administrado por FOSIS. }\end{array}$ & No \\
\hline & $\begin{array}{l}\text { Programa Caminos: dirigido a apoyar } \\
\text { a niños/as de familias con situaciones de } \\
\text { separación forzosa por cumplimiento de } \\
\text { condena, ejecutado por ONGs }\end{array}$ & No \\
\hline & $\begin{array}{l}\text { Programa Calle: orientado al trabajo } \\
\text { con adultos, ejecutado por municipali- } \\
\text { dades, Gobernaciones Provinciales y } \\
\text { ONG's. }\end{array}$ & No \\
\hline Chile Crece Contigo & $\begin{array}{l}\text { Sistema de Protección Integral a la In- } \\
\text { fancia, tiene como misión acompañar, } \\
\text { proteger y apoyar integralmente, a to- } \\
\text { dos los niños, niñas y sus familias, a tra- } \\
\text { vés de acciones y servicios de carácter } \\
\text { universal, focalizando apoyos especia- } \\
\text { les a los de mayor vulnerabilidad. }\end{array}$ & No \\
\hline \multirow[t]{3}{*}{$\begin{array}{r}\text { Oficina de Servicios } \\
\text { Relacionados: } \\
\text { CONADI: Promover, } \\
\text { coordinar y ejecutar } \\
\text { acciones para el desarro- } \\
\text { llo integral de personas } \\
\text { y comunidades indígenas. }\end{array}$} & $\begin{array}{l}\text { Fondo de Desarrollo: } 1 \text {. Manejo y Pro- } \\
\text { tección del Patrimonio Cultural indíge- } \\
\text { na; } 2 \text {. Subsidio a la capacitación y espe- } \\
\text { cialización indígena; } 3 \text {. Difusión y fo- } \\
\text { mento de las culturas indígenas; } 4 \text {. Apli- } \\
\text { cación del diseño curricular y pedagógi- } \\
\text { co intercultural bilingüe; } 5 \text {. Recuperación } \\
\text { y revitalización de las lenguas indígenas. }\end{array}$ & No \\
\hline & Fondo de Cultura y Educación. & No \\
\hline & Fondo de Tierra y Aguas indígenas. & No \\
\hline $\begin{array}{r}\text { INJUV: organismo } \\
\text { encargado de colaborar } \\
\text { con en el diseño, } \\
\text { planificación y coordina- } \\
\text { ción de políticas relativas } \\
\text { a los asuntos juveniles } \\
\text { (15-29 años). }\end{array}$ & $\begin{array}{l}\text { Desafío Clave: } \\
\text { Proyecto Huertos Urbanos Sociales } \\
\text { Proyecto Baños Integrales Sociales } \\
\text { Proyecto Importa } \\
\text { A mover Chile }\end{array}$ & $\begin{array}{l}\text { No } \\
\text { No } \\
\mathrm{Si}\end{array}$ \\
\hline SENADIS: & $\begin{array}{l}\text { Promover la igualdad de oportunidades, } \\
\text { inclusión social, participación y accesi- } \\
\text { bilidad de las personas con discapacidad. }\end{array}$ & No \\
\hline
\end{tabular}




\begin{tabular}{|c|c|c|}
\hline \multirow[t]{3}{*}{$\begin{array}{r}\text { FOSIS: apoya a } \\
\text { personas en situación de } \\
\text { pobreza o vulnerabilidad, } \\
\text { para mejorar su condi- } \\
\text { ción de vida. }\end{array}$} & $\begin{array}{l}\text { Emprendimiento } \\
\text { 1. Programa Yo Emprendo en Comuni- } \\
\text { dad; 2. Programa Yo Emprendo Semilla; } \\
\text { 3. Programa Yo Emprendo; 4. Programa } \\
\text { de Acceso al Crédito y 5. Programa Edu- } \\
\text { cación Financiera. }\end{array}$ & No \\
\hline & $\begin{array}{l}\text { Habilitación Social: } 1 \text {. Programa ac- } \\
\text { ción en familia; } 2 \text {. Programa acción en } \\
\text { comunidad; 3. Programa PUENTE; } 4 . \\
\text { Programa habitabilidad y 5. Programa } \\
\text { autoconsumo. }\end{array}$ & No \\
\hline & $\begin{array}{l}\text { Trabajo: 1. Programa yo trabajo y } 2 . \\
\text { Programa yo trabajo jóvenes }\end{array}$ & No \\
\hline \multirow[t]{6}{*}{$\begin{array}{r}\text { SENAMA: Fomentar el } \\
\text { envejecimiento activo y } \\
\text { el desarrollo de servicios } \\
\text { sociales para las perso- } \\
\text { nas mayores }\end{array}$} & $\begin{array}{l}\text { Fondo Nacional del Adulto Mayor: en- } \\
\text { trega recursos a organizaciones de per- } \\
\text { sonas mayores para desarrollo de pro- } \\
\text { yectos. }\end{array}$ & No \\
\hline & $\begin{array}{l}\text { Asesores Seniors: personas mayores } \\
\text { voluntarias le entreguen apoyo escolar a } \\
\text { niños y niñas que cursan entre } 1^{\circ} \text { y } 8^{\circ} \\
\text { año de educación básica, pertenecientes } \\
\text { a familias del Prog. Puente. }\end{array}$ & No \\
\hline & $\begin{array}{l}\text { Programa Vínculos: orientado al apo- } \\
\text { yo de adultos/as mayores vulnerables y } \\
\text { que viven solos. }\end{array}$ & No \\
\hline & $\begin{array}{l}\text { Turismo Social: financia viajes por un } \\
\text { día a lugares de interés histórico, cultural } \\
\text { y turístico. }\end{array}$ & No \\
\hline & $\begin{array}{l}\text { Servicios y vivienda para el Adulto } \\
\text { Mayor: brinda soluciones habitacionales } \\
\text { y servicios de apoyo a personas mayo- } \\
\text { res vulnerables. }\end{array}$ & No \\
\hline & $\begin{array}{l}\text { Escuelas de Formación para Dirigen- } \\
\text { tes de Organizaciones de Mayores. }\end{array}$ & No \\
\hline
\end{tabular}

Un segundo ministerio chequeado fue el MINVU, particularmente la Secretaría Ejecutiva de Desarrollo de Barrios, que contempla dos proyectos: Recuperación de Barrios y Condominios Sociales (cada uno con dos fondos a licitar). Así, de los 4 proyectos, sólo Recuperación de Barrios cumplió con los criterios de selección. Un tercer ministerio revisado fue el del Interior; en particular el Fondo Nacional de Seguridad Pública, donde se aloja el Programa de Gestión en Seguridad Ciudadana que a su vez contiene 
dos proyectos: los Proyectos de Intervención Social y el Proyecto Barrio en Paz, seleccionándose este último.

Un cuarto ministerio revisado fue de Medio Ambiente, a través del Fondo de Protección Ambiental, donde se ubican dos programas: el Concurso de Gestión Ambiental Local y el Programa Barrios Verdes. De esos dos programas revisados, ninguno fue seleccionado. El quinto ministerio fue el de Educación, que contiene 4 áreas: la Junta Nacional de Jardines Infantiles (JUNJI) con dos programas a su haber, el Programa de Mejoramiento de Atención a la Infancia (PMI) y el Programa de Jardines Comunitarios; la JUNAEB (Junta Nacional de Auxilio Escolar y Becas) y el CONICYT (Comisión Nacional de Ciencia y Tecnología) y DIBAM (Dirección Nacional de Bibliotecas, Archivos y Museos). Estas últimas 3 áreas no fueron revisadas por su pertinencia programática. A partir de la revisión, sólo se seleccionó el PMI.

Seguimos la revisión con el Consejo Nacional de la Cultura y las Artes (con rango ministerial), que contiene 4 fondos: el Fondo Nacional de Fomento del libro y La Lectura, el Fondo Nacional de Desarrollo de Cultura y Artes (FONDART), el Fondo de la Música Nacional y el Fondo Audiovisual. De estos 4 fondos revisados, seleccionamos el primero. También revisamos el Ministerio de Trabajo y Previsión Social, particularmente el Fondo para la Educación Previsional, pero no calificó para su inclusión. Para el caso del Ministerio de Secretaría General de Gobierno (SEGOB) revisamos dos fondos: el Fondo de Fortalecimiento de las Instituciones de Interés Público y el Fondo de Fomento de Medios de Comunicación Social regionales, provinciales y comunales; además del Instituto Nacional del Deporte (IND) que también depende de la SEGEGOB y que contiene al Fondeporte y el Proyecto Chile Estadios. De estos 4 proyectos, ninguno fue seleccionado.

En relación al Ministerio de Justicia, consideramos dos áreas: la primera es el Servicio Nacional de Menores, que a su vez, contiene 3 áreas específicas: el Área de Adopción (con 5 programas), el Área de Protección (que contiene a los 4 centros residenciales), los Programas de Diagnóstico y la línea de Programas que ejecutan las instituciones colaboradoras- con un total de 9 programas) y el área de Justicia juvenil (con 10 programas a su haber). La segunda área de dicho ministerio, corresponde a Gendarmería de Chile, que contiene a los establecimientos penitenciarios (5), a las Medidas Preventivas (1) y a los programas de Apoyo Postpenitenciario (3). En total para el SENAME registramos 29 programas, mientras que para Gendarmería 9, de los cuales sólo seleccionamos dos: el Programa de Prevención Comunitaria (PPC) y la Oficina de Protección de Derechos (OPD).

Finalmente, el Ministerio de Salud, que considera las siguientes divisiones: Prevención de Control de Enfermedades, Planificación Sanitaria, Finanzas y Administración Interna, Secretarías regionales ministeriales de salud, División de redes asistenciales y Políticas públicas y Promoción, que a su vez contiene al Departamento de Nutrición y Alimentos (con 4 programas), al Departamento de Promoción de la salud y participación ciudadana, 
al Departamento de Salud Ambiental y al Departamento de Políticas Farmacéuticas y Profesiones Médicas. De todos los programas, sólo calificaron dos: el Programa de Salud Integral para Adolescentes y Jóvenes y el Programa de Salud Mental y Psiquiatría.

Esta operación de aplicación de criterios comunitarios ejecutada, resultó en la selección de 9 programas que cumplían con los criterios propuestos, señalados en la tercera columna de la Tabla $\mathrm{N}^{\circ} 3$. Una vez revisados, aplicamos un tercer filtro para seleccionar aquellos programas que podrían constituirse en casos ejemplares de la inclusión de las dimensiones ético-políticas de la PC. El resultado de esta segunda fase fue la selección de 5 potenciales estudios de casos (destacado en la cuarta columna de la Tabla $\mathrm{N}^{\circ}$ 3): Programa de Mejoramiento de atención a la Infancia (PMI, Ministerio de Educación); Programa de Prevención Comunitaria (PPC, Ministerio de Justicia); Oficina de Protección de Derechos (OPD, Ministerio de Justicia); Recuperación de Barrios (Ministerio de Vivienda y Urbanismo) y Barrio en Paz (Ministerio Del Interior).

\section{Tabla No 3: Programas catastrados y programas fichados según Ministerio}

\begin{tabular}{|c|c|c|c|}
\hline Ministerio & $\mathrm{N}^{\circ}$ & Programas fichados & $\begin{array}{c}\text { Criterio } \\
\mathrm{s}\end{array}$ \\
\hline Ministerio de Desarrollo Social & 38 & A Mover Chile & 0 \\
\hline Ministerio de Vivienda Urbana & 4 & Recuperación de Barrios & 1 \\
\hline Ministerio del Interior & 2 & Barrio en Paz & 1 \\
\hline Ministerio del Medio Ambiente & 2 & 0 & 0 \\
\hline Ministerio de Educación & 2 & Programa Mejoramiento de la Infancia & 1 \\
\hline $\begin{array}{r}\text { Consejo Nacional de la Cultura y } \\
\text { las Artes }\end{array}$ & 4 & Fondo de fomento del libro y la lectura (*) & 0 \\
\hline $\begin{array}{r}\text { Ministerio del Trabajo y Previsión } \\
\text { Social }\end{array}$ & 1 & 0 & 0 \\
\hline Secretaría General de Gobierno & 4 & 0 & 0 \\
\hline Ministerio de Justicia & 38 & $\begin{array}{l}\text { Oficinas de Protección de Derechos del niño, niña } \\
\text { y adolescente(OPD) }\end{array}$ & 2 \\
\hline Ministerio de Salud & 6 & $\begin{array}{l}\text { Programa de salud integral para adolescentes y } \\
\text { jóvenes }(*) \\
\text { Programa Nacional de Salud Mental (*) }\end{array}$ & 0 \\
\hline Total & 101 & 9 & 5 \\
\hline
\end{tabular}

$\left(^{*}\right)$ No cumplen con todos los criterios, fueron eliminados en el tercer filtro

En esta Tabla se destaca, en primer lugar, que la mayor cantidad de programas aparecen instalados en los Ministerios de Desarrollo Social y de Justicia; 38 en cada uno, lo que corresponde al 75\% de los programas; tal focalización parece coherente con los énfasis y prioridades establecidas por el gobierno de Sebastián Piñera, quien privilegió en su programa de gobierno la disminución de la delincuencia y de la pobreza. Asimismo, llama la atención la exigua cantidad de programas instalados en el Ministerio de Salud, y particularmente, que ninguno de ellos contenga o contemple una perspectiva comunitaria. 


\section{Una mirada a tres programas comunitarios, ejemplares en la política social chilena}

En la tercera y última fase, seleccionamos 3 programas considerando tanto la accesibilidad de los contactos como que su ejecución fuese tanto en la Región Metropolitana como en la Región de Valparaíso: Recuperación de Barrio (RB), perteneciente al MINVU; el Programa de Prevención Comunitaria (PPC) del SENAME y el Programa de Mejoramiento de Atención a la Infancia (PMI) de JUNJI. A partir del análisis de documentos y entrevistas que realizamos a diseñadores, ejecutores y usuarios/as de los programas y, como se aprecia en la Figura $\mathrm{N}^{\mathrm{o}}$ 1, se identificó tres categorías centrales que contienen los aspectos compartidos (o comunes) por los programas estudiados: dimensión ética, dimensión política y dimensión metodológica.

\section{Figura $N^{0}$ 1: Categorías emergentes en el Análisis de Caso}

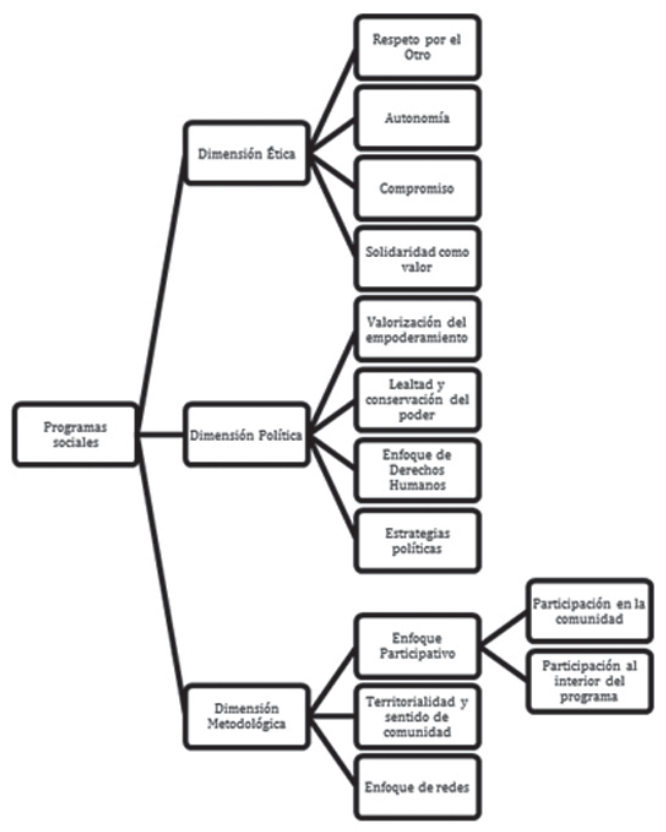

La dimensión ética emerge de los relatos de las/os entrevistadas/os cuando se refieren a la noción de lo correcto, deseado y valorado por el programa para la organización y la comunidad. El respeto por el otro se constituye en un principio ético central, que incorpora un reconocimiento y valor a los aportes de los distintos agentes de la comunidad y que subyace a algunos objetivos que se privilegian en las prácticas y actividades pro- 
gramadas con ella. Por ejemplo, el reconocimiento de los humanos "como iguales” posibilita generar instancias reales de participación, mientras que en otros casos observamos que este principio, aporta transformar la visión de la comunidad logrando respetar su trabajo:

“Entonces para mí relevar la participación como el foco central del programa es como clave para entender desde lo ético, para entender al ser humano y para abrir las posibilidades que todos tenemos de ser iguales, como ya generemos esta oportunidad que discutamos al mismo nivel”. (Diseñadora PPC, 2013)

"Yo diría así intuitivamente que con los PMI se logró transformar la visión de la escuela hacia los párvulos. La escuela siempre miraba al párvulo como incapaz, como que los niños aprenden solamente cuando están conmigo. Las educadoras decíamos "los niños, cuando estamos con el delantal verde, aprenden”. Yo me acuerdo muy emocionada de una directora de una escuela en Buin; de repente se empieza a dar cuenta que están pasando cosas súper buenas. Entonces se compromete cien por ciento. El PMI era su chiche y sufre toda una transformación desde su visión, como desde "qué bueno que vengan a jugar” a "pucha qué maravilla ¡cómo están aprendiendo!”. (Diseñadora PMI, 2013)

El respeto se traduce también en la valoración de la autonomía, tanto personal como comunitaria. En esta dimensión se describe, como punto de partida, el reconocimiento de la comunidad como Otro válido y con capacidades para hacerse cargo de su propio desarrollo. Refiere a la importancia de validar el progreso de las comunidades desde un horizonte de reconocimiento y respeto, donde todas las posibilidades de ser y actuar son legítimas. El compromiso del Estado con la comunidad en el marco de su rol "protagonista” en la construcción de la intervención:

"O sea, y fuimos entonces dosificando los apoyos: primer año, qué es lo que más necesitan, el segundo año, qué es lo que más necesitan y el tercer año y ya a partir del cuarto año autonomía, mucha más autonomía porque ya estaban como maduros (...) Entonces también fuimos aprendiendo eso en el camino...”. (Diseñadora PMI, 2013)

"También darle la posibilidad al otro que, de que sea sujeto y eso implica que te puede rechazar, que te puede [decir] no quiero que se meta y un montón de cosas. (Diseñadora PPC, 2013).

Encontramos que el valor del Compromiso es el principal componente de la relación de sus integrantes con la organización y refiere a intereses comunes, pero principalmente a ciertas responsabilidades asumidas y compartidas:

"Mucha solidaridad entre ellas. Las capacitaciones iban mucho de la mano de la colaboración, aprender en colaboración. Bueno y asumir 
la responsabilidad, también habría otro valor, asumir que lo que están haciendo tiene que ser responsable. No puede ser que si dicen que van a funcionar lunes y martes, después no lleguen. No... nunca nos pasó pero estaba como implícito que tenías que ser responsable con tus compromisos. Por ahí yo diría que van más bien los valores... que estaban... que los trabajábamos...”. (Diseñadora PMI, 2013)

Finalmente, la Solidaridad como valor se constituye en un contenido de la dimensión ética que incide en el funcionamiento de la organización y se valida particularmente a través del apoyo mutuo o el poder contar con un Otro para el abordaje de los problemas. En este sentido observamos como muchas veces, la ayuda mutua y la solidaridad aparecen como complemento o incluso a veces como respuestas contingentes frente a la indiferencia y la tendencia del Estado a eludir su responsabilidad:

“Acá tenemos varias organizaciones que nos apoyan, nos están mandando siempre cosas, está grupo de danza, un grupo de viejitos que son poetas y escritores de aquí abajo y siempre nos están aportando cosas y gente que viene a vernos y esas son las redes; con el consultorio igual, con la casa de la familia y la iglesia que nos presta el espacio, porque este espacio no es propio.” (Ejecutora 2 PMI, 2013)

"Ese es un aprendizaje que el programa lo ha ido desarrollando en términos generales, desde la línea central, desde la secretaría ejecutiva, desde el aporte de las regiones, en fin. Bueno y también uno a nivel regional de alguna forma trata de ir incorporando o de ir recopilando ciertos aprendizajes o ciertas formas de cómo el programa opera, o cuales han sido los aciertos y cuáles han sido los errores, o cuáles han sido las deficiencias del programa, para irlas incorporando.”(Ejecutora, RB, 2013)

La dimensión política refiere a la noción de poder que se pone en juego al momento de comprender la participación de los distintos actores en el programa; lo que deriva en el reconocimiento de su capacidad para opinar y decidir sobre los asuntos que afectan su desarrollo en un marco de lealtad y conservación del poder. En la concepción de la organización y de las actividades prevalece el interés por fomentar un aumento del poder de las personas y comunidades, mostrándose, de este modo, una especial valoración del empoderamiento.

"Entonces ahí uno dice, pucha, ahí tú ves el poder, cómo la gente cuando tú le das autonomía, le das responsabilidades, crees en ellos, confías en ellos, los acompañas en sus proyectos, las gente se empodera, se desarrolla, se despliega. Se despliega, era cuestión de ver, no sé poh', darle un mínimo de posibilidades para que como que la gente florecía (...) Entonces, uno veía una movilización ahí muy linda, muy linda”... (Diseñadora PMI, 2013). 
"Y a raíz del terremoto yo tuve que aprender. Entonces habíamos varios dirigentes que ya manejábamos los temas de... estudios de... ¿cómo te dijera? Interpretar los planos, las mecánicas del suelo, las calicatas, todos esos términos ya los manejamos. Sabemos cuándo un terreno es apto para construir, no es apto para construir, hemos aprendido una gama de cosas... Y lindo, porque tú vas aprendiendo, y después tú cuando vas al programa del consejo, tú das tu experiencia, y cuando los profesionales te van explicando, tú vas preguntando ya cosas más técnicas”. (Usuaria RM, 2013 ).

Desde un perspectiva consistente con el Enfoque de derechos humanos, se asume que la intervención debería ayudar a la redistribución de poder al interior de las comunidades y en el establecimiento de relaciones horizontales al interior de la sociedad.

"Valores yo diría que nuestro... este proyecto era centralmente valórico, valórico total. Si me dijera cuál es la plataforma, está en lo valórico. Primero en reconocer los seres humanos independientemente de si es pobre o es rico, da lo mismo, es ser humano, sujeto de derecho central desde que nace: derecho a que sus padres o madre o familia los eduquen, derecho a que el Estado los acompañe, que ofrezca las oportunidades y bueno... y todos los valores más explícitos que fomentábamos en el PMI: es respeto por el niño, todas las decisiones tenían que pasar porque era beneficioso para los niño”. (Diseñadora PMI, 2013).

"Yo creo que primero el tema de los derechos, o sea entender que partimos del entendimiento que todos somos sujetos de derechos y que al mismo tiempo hay un Estado que tiene que ser garante y que el resto de seres humanos mortales digamos también estamos, somos co-garantes desde sus derechos y a nosotros nos compete un rol en eso y yo creo que eso que tiene que ver como con los derechos y las responsabilidades que tenemos para abrir oportunidades pa' que se ejerzan esos derechos también de los distintos actores” (Diseñadora PPC, 2013).

Respecto de las estrategias políticas implicadas en la formulación, desarrollo y ejecución de los programas, observamos que en un nivel más específico emergen intereses y acciones orientadas a su legitimación (dentro de distintas organizaciones) y, al mismo tiempo, posibilitar su continuidad, neutralizando distintas amenazas, algunas que derivan incluso del mismo sistema público. Por ejemplo, la necesidad de mostrar la efectividad de los programas a través de datos "duros” para asegurar su continuidad; o acciones de difusión a la prensa y al sistema a través de “actos públicos” que lo visibilizan, así como manifestaciones de la propia comunidad en defensa y apoyo de los programas.

“Entonces decidimos hacer una gran jornada nacional PMI-CASH, juntamos a no sé cuántas miles de personas aquí en Santiago y le 
contamos la firme: lo que quiere el Ministerio de Educación es que ustedes se vayan para su casa y se las arreglen con fondos propios. Pa’ qué te digo: senadores, diputados, alcaldes... funcionó la máquina y el ministerio tuvo que echar marcha atrás. Y tanto es así que el jefe que es el ideólogo de esta cuestión, reconoció formalmente en una reunión que le habíamos ganado” (Diseñadora PMI, 2013).

“Entonces por eso es que la sociedad civil es súper importante porque la sociedad civil es la que está en los territorios y la que tiene la experticia metodológica para trabajar la participación y ciudadanía y por eso por ejemplo ahora, estamos haciendo muchas cosas en conjunto con los programas de intervención comunitaria y la protección de derechos”. (Diseñadora PPC, 2013)

Finalmente, con respecto a la dimensión metodológica que reúne los contenidos asociados a los componentes técnicos que están a la base del diseño e implementación de los programas, observamos una directa relación con las propuestas de la PC. El Enfoque participativo se constituye en uno de los ejes metodológicos fundamentales en los casos estudiados y comprende distintos niveles, como son la participación en la comunidad, junto a distintos actores y la participación al interior del programa mismo. La primera refiere a aquellos elementos que conforman el núcleo metodológico que articula las principales estrategias con las que se diseña e implementa el modelo. Así, se destaca la importancia de la participación de distintos actores para promover espacios de co-construcción y colaboración comunitaria.

“La idea del programa, más que la idea, el propósito es renovar o incentivar la participación comunitaria no sólo en el consejo vecinal de desarrollo, sino que las propias organizaciones que participan en el consejo sigan participando, y es como incentivar esa participación también.” (Ejecutora RB, 2013)

“El modelo de los PPC, involucra en su ejecución a distintos actores: niños, niñas y adolescentes, sus familias y actores comunitarios. Implementando con cada uno de ellos metodologías particulares a sus características y al aporte que se espera realicen en la instalación participativa de dispositivos de promoción y protección de derechos” (Documento EPPPC, PPC, 2011)

La Participación al interior del programa tiene distintos grados y va desde la sola asistencia de los participantes o sus familias a convocatorias específicas, hasta un grado más profundo, relacionado con la posibilidad de opinar y tomar decisiones sobre actividades o cambios al interior del programa.

"Se considera que el programa es en esencia un programa participativo, que considera la participación comunitaria como un eje fundamental, más no es un eje fundamental está en la génesis del 
programa la participación de la comunidad, o sea, como que se explicita la transversalización de ese” (Ejecutora RB, 2013)

“Algunos papás asisten, otros no. Igual debo decir que la participación es súper baja de parte de las familias y siempre uno cuenta con los claves que son los que están ahí siempre, y tú sabes dónde están y que tienen una buena recepción. Hemos incluido, por ejemplo, los encuentros que hacemos con las mamás que siempre que saben, sabemos que tenemos más llegada, las invitamos a los encuentros que hacemos deportivos. También invitamos a familias, participan también” (Ejecutora PPC, 2013)

Son también relevantes, en la perspectiva de asegurar la calidad de la intervención, los adecuados procesos de familiarización y de inserción territorial, que permitan respetar y preservar el sentido de pertenencia, en su dimensión más simbólica que sólo física. En este sentido, identificamos el sentido comunidad como un aspecto que está intrínsecamente vinculado con la noción de territorialidad.

“A ver, yo creo que ahí lo diferenciaría: institucionalmente, yo creo que es donde ha sido más radical y tal vez al principio lo teníamos en el discurso, pero yo creo que ahora ya lo tenemos como mucho más claro, es intervenciones territoriales. Eh, el gran cambio es que llegamos y queremos mirar el territorio, no queremos mirar vivienda para una familia, una calle para un comité; no, es un territorio que queremos tratar de abordad integralmente” (Diseñadora RB, 2013)

"Por eso nosotros hablábamos de los micro territorios, que fueran barrios, que tuviera como pertinencia, o sea que no fuera un, ojalá como que la apuesta que no sea como una delimitación así como hecha en el mapa a lápiz, sino que sea la población "X”, el sector tanto que se tenga como un referente para la comunidad” (Diseñadora PPC, 2013)

\section{Discusión y Conclusiones}

En este artículo presentamos resultados cuantitativos y cualitativos que, al ser contrapuestos, muestran un panorama muy contradictorio en cuanto al lugar que juegan conceptos, valores y principios de la PC en las políticas sociales chilenas.

Cuantitativamente, como se demuestra en el catastro de 101 programas/proyectos, de 10 ministerios nacionales examinados, contienen una escasa perspectiva comunitaria o consideración de los principios y prácticas de la PC. Sólo nueve programas cumplieron con los criterios que establecimos para considerarlos "comunitarios". Nos parece evidente que para intentar comprender esta exigua consideración de la PC en la política pública chilena, debemos recurrir al contexto socio-político en que se desarrolló 
este estudio. El catastro puede ser considerado una "fotografía” que da cuenta de las formas en que el gobierno, durante el último año de la presidencia de Sebastián Piñera, efectivamente concibió la política social. Y esta "fotografía”, en términos cuantitativos, muestra lo que podríamos denominar una involución en el desarrollo de la disciplina en el ámbito público.

En contraposición a lo descrito para la década del 90, cuando se reportaba una cierta masificación de la inscripción de la PC en programas sociales (Alfaro \& Zambrano, 2009; Asún \& Unger, 2007), encontramos ahora una franca ausencia, omisión o exclusión de conceptos, principios, valores y prácticas comunitarias. Así, respecto al ejemplo más extensamente descrito en este artículo, la oferta programática del Ministerio de Desarrollo Social, encontramos que de 33 programas ofertados en este ministerio -que por sus objetivos se esperaría fuese espacio privilegiado para los aportes de la PC-, sólo uno de ellos puede ser considerado "comunitario".

Cabe señalar que a nivel del discurso, en los nombres de los programas, su descripción, en los objetivos, o en general en las bases técnicas, es fácil encontrar la aplicación de conceptos, teorías, principios y propuestas propias de la PC. Es así que encontramos como objetivos declarados, la generación de condiciones socio comunitarias que favorezcan que las personas y familias puedan revertir y generar cambios en su condición social de pobreza y exclusión, tales como, por ejemplo, desarrollar capacidades propias, autonomía, derechos ciudadanos y voz pública; objetivos centrados en el desarrollo de control cultural de las personas en condición de pobreza sobre su propia vida. Ello se aprecia claramente en SENAMA que como institución se plantea la misión de: "Fomentar el envejecimiento activo y el desarrollo de servicios sociales para las personas mayores, cualquiera sea su condición, fortaleciendo su participación y valoración en la sociedad, promoviendo su autocuidado y autonomía, y favoreciendo el reconocimiento y ejercicio de sus derechos". No obstante, los programas que ofrece poseen un marcado énfasis asistencialista, centrado en las personas (no en la comunidad) y en la satisfacción de necesidades básicas, como el de Turismo Social o el Servicio de Vivienda para el Adulto Mayor.

Esta discordancia entre discurso y práctica genera ilusiones peligrosas, tanto para los/as usuarios/as de los programas, como para las y los profesionales que se desempeñan en el campo de los programas sociales. Emerge así la pregunta acerca del rol que los/as profesionales psicólogos/ as poseen actualmente en su inserción en programas estatales que son presentados como comunitarios.

Los datos numéricos contradicen la variada experiencia reportada en el marco teórico de este trabajo, en que se describe la riqueza potencial y real de los aportes de la PC a las políticas públicas en distintos países. Se estarían perdiendo grandes posibilidades que la PC ofrece de enriquecer y nutrir las políticas públicas, al focalizar los programas sociales a través de perspectivas individualistas y asistencialista de corte neoliberal. Es así como, 
estos resultados globales del catastro pueden ser considerados, en cierto sentido, coincidentes con el reporte de Alfaro \& Zambrano (2009), cuando señalan que la política social del último período en Chile conlleva un reduccionismo que circunscribe la responsabilidad pública a políticas focalizadas en los pobres, enfatiza intervenciones a nivel de los individuos o las familias y deja fuera de las orientaciones de la política social dimensiones de ciudadanía y el bienestar general de la población.

Y, en forma igualmente dramática, los resultados de este estudio se muestran coherentes con lo reportado por Reyes (2007), cuando señala que la mayor parte de las publicaciones comunitarias entre 1993 y 2003 en Chile sustentan principios y valores de tipo Asistencial-Consultivo (54\%): orientaciones programáticas que, haciendo una valoración de los participantes en el proceso, promueve acciones de tipo "curativas", es decir, focalizadas en la solución de los problemas que aquejan a los grupos de riesgo o población objetivo. Asimismo, señala que, en general, las orientaciones éticovalóricas de psicología comunitaria nacional de los últimos años se traducen en acciones que relegan a un plano secundario la consideración de aspectos promocionales y transformadores en la comunidad ya que enfatizan un polo negativo, carencial y deficitario. Es decir, en los únicos tres estudios que reportan datos cuantitativos, el de Reyes (2007), el de Berroeta, Hatibovic \& Asún (2012) y el que presentamos en este artículo, encontramos un panorama en que no se aprecia el aporte de la PC a la política pública, como se esperaría a partir del grado de desarrollo que la disciplina posee en el país.

Ahora bien, se contrapone a este panorama más bien pesimista, la existencia de excepciones, muy débiles numéricamente, pero poderosas en su significado, que revelan que algunos programas gubernamentales han logrado incorporar algunas contribuciones provenientes de la disciplina, ampliando el alcance de las intervenciones en el trabajo directo con las comunidades. Este hecho ya ha sido reportado por diversos autores en los últimos años quienes insisten en establecer puentes de colaboración mutua entre la PC y las políticas sociales (Montero, 2009; Rodríguez, 2012; Nelson, 2013; Alfaro, 2013; Wiesenfeld, 2014).

Algunos de los ejemplos que destacamos en esta investigación corresponden los programas PMI, PPC y RB, que incluyen en su diseño e implementación elementos básicos del encuadre comunitario, como es la utilización de la noción de comunidad, relevando su dimensión territorial en tanto una dimensión necesaria para la construcción de sujetos situados (Figueroa, 2012)-, la que permite contar con dinámicas relacionales entre distintos actores sociales que se potencian a partir de la acción programática ${ }^{3}$. Más relevadora aún de manifestaciones concretas del aporte de la PC a las políticas públicas es la forma en que aprecian las dimensiones ético-políticas en los casos en que un determinado programa o proyecto efectivamente posee una orientación comunitaria. Es así como, encontramos valores que han sido descritos como propios de la PC, tanto en el diseño como en la implementación de algunos de los programas que mencionamos como 
excepciones en el catastro: respeto por el Otro, autonomía, compromiso y solidaridad. Esta dimensión ética fundamenta lo que es considerado correcto o incorrecto en los programas, y se relaciona estrechamente con la dimensión política, que enfatiza la valoración del empoderamiento y la aplicación del enfoque de derechos en algunos de los programas analizados.

Este contraste entre los resultados cuantitativos -ausencia de perspectiva comunitaria- y los resultados cualitativos -riqueza de aportes éticos y políticos de la PC- da cuenta de un campo de posibilidades que no se está aprovechando. Constituye un desafío pendiente, entonces, encontrar las formas en que la PC puede efectivamente tener una presencia significativa y nutrir desde su saber y quehacer a los programas sociales y las políticas públicas en nuestro país.

Al respecto, llama la atención que entre aquellos sectores de la política pública que se muestran más resistentes a la perspectiva de la PC, están el ámbito de la salud y el de cultura, los que en distintos momentos de la historia de la política pública chilena, habían manifestado innovaciones técnicas interesantes. Hoy no cuentan con dispositivos técnicos que hagan posible el trabajo con y desde las comunidades.

Por último, es relevante destacar que aun cuando se evidencian las contradicciones antes señaladas, en lo que respecta al diseño e implementación de los programas, sí existe una correspondencia a nivel metodológico de las orientaciones propias de la PC, reconociéndose la inclusión de elementos como el Enfoque Participativo, el Sentido de Comunidad, la Territorialidad y el Fortalecimiento. No obstante, creemos que es prudente mirar con cautela esta relación, en tanto puede ser interpretado como un indicador que da cuenta más de una instrumentalización, que de un legítimo reconocimiento. 


\section{Notas}

${ }^{1}$ Esta publicación reporta resultados del proyecto de investigación FONDECYT $\mathrm{N}^{\circ}$ 113638: Ética y Política ¿Dimensiones olvidadas en la Psicología Comunitaria hoy?

${ }^{2}$ Información extraída en abril del año 2013; desde la página web del ministerio de Desarrollo Social y de la página www.participemos.gob.cl que registraba los fondos concursables por ministerio.

${ }^{3}$ Mientras este artículo está en revisión, el SENAME decide terminar con los PPC en Enero de 2015, argumentando la necesidad de reemplazarlos por un sistema de "mayor cobertura” e ignorando diversos estudios que avalan su impacto y efectividad. 


\section{Bibliografía}

Alfaro, J. (2012), "Posibilidades y tensiones en la relación entre Psicología Comunitaria y políticas sociales”. En Alfaro, J., Sánchez, A. y Zambrano, A. (Eds.), Psicología Comunitaria y Políticas Sociales. Reflexiones y experiencias (pp. 45- 75), Paidós, Buenos Aires.

Ídem (2013), "Psicología Comunitaria y Políticas Sociales: Institucionalidad y dinámicas de actores”. Global Journal of Community Psychology Practice. Recuperado de: http://www.gjcpp.org/pdfs/alfaro-v4i2-20130613.pdf

Alfaro, J. \& Zambrano, A. (2009), "Psicología Comunitaria y políticas sociales en Chile”. Revista Psicología \& Sociedade, 21(2), 275-282.

Arrieta, C. \& Garita, C. (2012). “Construcción de política social: retos para la Psicología Comunitaria costarricense de cara a los procesos de formación profesional y a la definición del papel psicológico comunitario”. En J. Alfaro, A. Sánchez y A. Zambrano (Eds.), Psicología Comunitaria y Políticas Sociales. Reflexiones y experiencias (pp. 359-379). Paidós, Buenos Aires.

Asún, D. \& Unger, J. (2007), “Una visión regional de la institucionalización de la psicología (social) comunitaria en Chile”. En A. Zambrano, G. Rozas, I. Magaña, D. Asún y R. Pérez-Luco (Eds). Psicología Comunitaria en Chile: evolución, perspectivas y proyecciones (pp. 213-226), RIL, Santiago de Chile.

Banyard, V. \& Miller, K. (1998), "The powerful potencial of qualitative research for community psychology". American Journal of Community Psychology 26(4), 485-505.

Berroeta, H. (2014), “El quehacer de la Psicología Comunitaria: coordenadas para una cartografía”. Psicoperspectivas, 14(2), 19-31.

Berroeta Torres, H., Hatibovic Diaz, F. \& Asún Salazar, D. (2012), "Psicología Comunitaria: prácticas en Valparaíso y visión disciplinar de los académicos nacionales”. Polis, Revista Latinoamericana, 11(31), 335-354.

Brady, S., Schoeneman A. \& Sawyer, J. (2014), “Critiquing and Analyzing the Effects of Neoliberalism on Community Organizing: Implications and Recommendations for Practitioners and Educators". Journal for Social Action in Counseling and Psychology, 6(1), 36-60.

Carvallo-Messa, V. \& Serrano-García, I. (2008), “¿Deben participar los psicólogos y psicólogas en política pública?: una mirada desde la psicología social-comunitaria”. Revista Psicol. Am. Lat. [online] 12. Recuperado en 16 de octubre de 2013, de http://pepsic.bvsalud.org/scielo.php?script=sci arttext\&pid=S1870-350X2008000100003\&lng=es\&tlng=es. 
Delamaza, G. (2011), “Espacio público y participación ciudadana en la gestión pública en Chile: límites y posibilidades”. Polis, Revista Latinoamericana, 10(30), 45-75.

Denzin, N. \& Linconl, Y. (1994), “Introduction. Entering the field of cualitative research”. En N. Denzin y I. Lincoln (Eds.), Handbook of qualitative research (pp. 1-17), Sage Publications, Thousand Oacks.

Figueroa, N. (2012), “El desarrollo y las políticas públicas”. Polis, Revista Latinoamericana, 11(33), 375-391.

Flores, R. (2010). Observando Observadores, Ediciones UC, Santiago de Chile.

Fuenmayor, J. (2014), Política pública en América Latina en un contexto neoliberal: Una revisión crítica de sus enfoques, teorías y modelos. Cinta de Moebio, 50, 39-52.

Glaser, B. \& Strauss, A. (1967), The Discovery of Grounded Theory, Aldine Publishing Company, Chicago.

González Rey, F. (2009), “Epistemología y Ontología: un debate necesario para la psicología de hoy”. Revista Diversitas Perspectivas en Psicología, 5(2), 205-224.

Hasenfeld, Y. \& Garrow, E. (2012), “Nonprofit Human-Service Organizations, Social Rights and Advocacy in Neoliberal Welfare State”. Social Service Review, 86(2), 295-322.

Iñiguez, L. (1999), “Investigación y evaluación cualitativa: bases teóricas y conceptuales”. Revista de Atención Primaria, 23(8), 496- 502.

Krause, M., Jaramillo, A., Monreal, V., Carvacho, H. \& Torres, H. (2011), "Historia de la Psicología Comunitaria en Chile: desde la clandestinidad a la política pública”. En M. Montero e I. Serrano-García (Eds.), Historias de la Psicología Comunitaria en América Latina. Participación y Transformación (pp. 115-138). Paidós, Buenos Aires.

Lapalma, A. \& de Lellis, M. (2012), “Psicología Comunitaria y políticas públicas: una articulación posible y necesaria”. En J. Alfaro, A. Sánchez \& A. Zambrano (Eds.), Psicología Comunitaria y Políticas Sociales. Reflexiones y experiencias (pp. 147-172), Paidós, Buenos Aires.

Lorion, R. (1996), Psychology and Public Policy: Balancing Public Service and Professional Need, APA, Washington.

Miles, M. B. \& Huberman, A. (1994), Qualitative data analysis: an expanded sourcebook, Sage, Newbury Park, CA. 
Montero, M. (2009), “Community Action and Research as Citizenship Construction”. American Journal of Community Psychology, 43, 149- 161. DOI 10.1007/s10464-008-9224-6

Ídem (2010), “Unión de actores sociales, participación comunitaria y ética, en la ejecución de políticas públicas”. En A. Hincapié, Sujetos políticos y acción comunitaria. Claves para una praxis de la psicología social y de la clínica social-comunitaria en América Latina (pp. 45-66), Universidad Pontificia Bolivariana, Medellín.

Nelson, G. (2013), “Community Psychology and Transformative Policy Change in the Neo-liberal Era”. American Journal of Community Psychology, 52(3-4), 211-223 DOI 10.1007/s10464-013-9591-5

Ornelas, J., Vargas-Moniz, M., \& Duarte, T. (2010), “Community Psychology and Social Change: A Story from field of Mental Heath in Portugal”. Global Journal of Community Psychology Practice, 1(1), 21-31.

Quintal de Freitas, F. (2000), "Voices from the South: The Construction of Brazilian Community Social Psychology”. Journal of Community \& Applied Social Psychology, 10, 315-326.

Reyes, M. I. (2007), “Orientaciones ético-valóricas de la Psicología Comunitaria en Chile: análisis descriptivo de los artículos publicados en revistas nacionales entre 1993 y 2003”. En J. Alfaro y H. Berroeta (Eds.), Trayectoria de la Psicología Comunitaria en Chile. Prácticas y Conceptos (pp. 9148), Editorial Universidad de Valparaíso, Valparaíso.

Rodríguez, A. (2012), “Aportes de la psicología comunitaria al campo de las políticas públicas sociales: el caso de Uruguay”. En J. Alfaro, A. Sánchez \& A. Zambrano (Eds.), Psicología Comunitaria y Políticas Sociales. Reflexiones y experiencias (pp. 111-142), Paidós, Buenos Aires.

Saavedra, C. (2005), Aproximación al desarrollo actual de la psicología comunitaria, desde el análisis de las prácticas que ésta construye en el campo de la intervención social. Tesis de Magíster en Psicología Comunitaria, Universidad de Chile, Santiago, Chile

Shinn, M. (2007), "Waltzing with a Monster: Bringing Research to Bear on Public Policy”. Journal of Social Issues, 63(1), 215-231.

Stein, C. \& Mankowski, E. (2004), “Asking, witnessing, interpreting, knowing: conducting qualitative research in community psychology”. American Journal of Community Psychology, 33(1-2), 21-35

Winkler, M.I.; Alvear, K.; Olivares, B. \& Pasmanik, D. (2009), Autonomía versus Dependencia. Quehacer comunitario y Políticas Públicas, Universidad de Santiago de Chile, Santiago. 
Ídem (2014), "Psicología comunitaria hoy: Orientaciones éticas para la acción”. Psicoperspectivas, 13(2), 44-54.

Wiesenfeld, E. (2014), “La Psicología Social Comunitaria En América Latina: ¿Consolidación o Crisis?”. Psicoperspectivas, 13(2), 6-18.

Recibido: 15.03.2014

Aceptado: 13.12.2015 